\title{
Photocatalytic degradation of MB from water by using Ni-TPPS/NiO nanoparticles
}

\author{
Rahmatollah Rahimi, Mohammad Bozorgpour, Mahboubeh Rabbani \\ Department of Chemistry, Iran University of Science and Technology, Narmak, Tehran 16846- \\ 13114, Iran \\ E-mail: Rahimi_rah@iust.ac.ir
}

$\mathrm{NiO}$ nanoparticles were synthesized by a sol-gel technique. $\mathrm{Ni}\left(\mathrm{NO}_{3}\right)_{2} \cdot 6 \mathrm{H}_{2} \mathrm{O}$ and citric acid were employed as starting raw materials. Then, the obtain result calcined at $550 \mathrm{OC}$ for $5 \mathrm{~h}$. The phase composition and the structure of the calcined products were investigated by X-ray diffraction and scanning electron microscopy techniques, respectively. XRD patterns exhibit six (111), (200), (220), (311), (222) and (400) characteristic peaks of cubic crystalline NiO. Then The Niporphyrin immobilized on surface of $\mathrm{NiO}$ and the potential of the obtained photocatalysts in degradation of methylene blue dye was studied under visible irradiation.

Keyworsds: NiO, photocatalyst, Methylene blue, Ni-TPPS

\section{Introduction}

One of the advantages of an inorganic matrix for embedding of functional chromophores is attributed to the more rigid stability and strongly increased photostability compared to organic matrix [1]. Organic photocatalysts of chlorophyll and porphyrin derivatives act under visible light illumination. They are well known for their biological conduction and photoactive properties and are very useful as catalysts[2-8]. Porphyrin like Chl-a absorbs light due to chelation of conjugated double bonds of chlorin ring with Mg (II) [9].

In this work, we report the synthesis of a new organic- inorganic hybrid optical material with nickel oxide and Ni-TPPS as inorganic and organic component, respectively. They form NiTPPS/NiO with a high photcatalytic activity.

\section{Experimental}

\subsection{Materials and Methods}


All of the Chemicals used in this work were analytical grade reagents and used without further purification. Nickel nitrate $\left(\mathrm{Ni}\left(\mathrm{NO}_{3}\right)_{2} \cdot 6 \mathrm{H}_{2} \mathrm{O}\right)$, citric acid, pyrrole, propionic acid, benzaldehyde, DMF and sulfuric acid were purchased from Merck company. Deionized water was used to prepare all solutions.

The samples were characterized by X-ray powder diffraction (XRD) using JEOL X-ray diffractometer with $\mathrm{Cu} \mathrm{Ka}$ radiation. The particle morphologies of the $\mathrm{NiO}$ powder were observed by an AIS2100 (Seron Technology) scanning electron microscopy (SEM). The FT-IR analyses were carried out on a Shimadzu FTIR-8400S spectrophotometer using a $\mathrm{KBr}$ pellet for sample preparation. DRS spectra were prepared via a Shimadzu (MPC-2200) spectrophotometer.

\subsection{Preparation of nickel meso-tetrakis(4-sulfonatophenyl) porphyrin (TPPS)}

$10 \mathrm{mmol}$ of freshly distilled pyrrole $(0.7 \mathrm{ml}), 10 \mathrm{mmol}$ of benzaldehyde, $100 \mathrm{ml}$ of propionic acid and $15 \mathrm{ml}$ of nitrobenzene were added to a $250 \mathrm{~mL}$ flask. The mixture was allowed to reflux under stirring at $120{ }^{\circ} \mathrm{C}$ for $60 \mathrm{~min}$. After that, the resulting mixture was cooled overnight in room temperature and filtrated under reduced pressure.

For synthesis of meso-tetrakis(4-sulfonatophenyl) porphyrin (TPPS), $0.2 \mathrm{~g}$ of TPP was dissolved in $5 \mathrm{ml}$ of concentrated $\mathrm{H}_{2} \mathrm{SO}_{4}$ (Scheme 1). The mixture was heated in a steam bath for $4 \mathrm{~h}$ then allowed to stand for $48 \mathrm{~h}$. The resulting solution was neutralized by $\mathrm{NaOH}(3 \mathrm{~N})$ and then the solvent was removed under vacuum at room temperature. The product was washed with methanol until no TPPS could be detected in the solution by UV-visible spectrophotometer.

For synthesis of nickel meso-tetrakis(4-sulfonatophenyl) porphyrin (Ni-TPPS), 1mmol of TPPS and $2 \mathrm{mmol} \mathrm{Ni}\left(\mathrm{NO}_{3}\right)_{2} \cdot 6 \mathrm{H}_{2} \mathrm{O}$ was dissolved in $20 \mathrm{ml}$ of DMF. The mixture was refluxed for $4 \mathrm{~h}$ then allowed to stand for $48 \mathrm{~h}$.

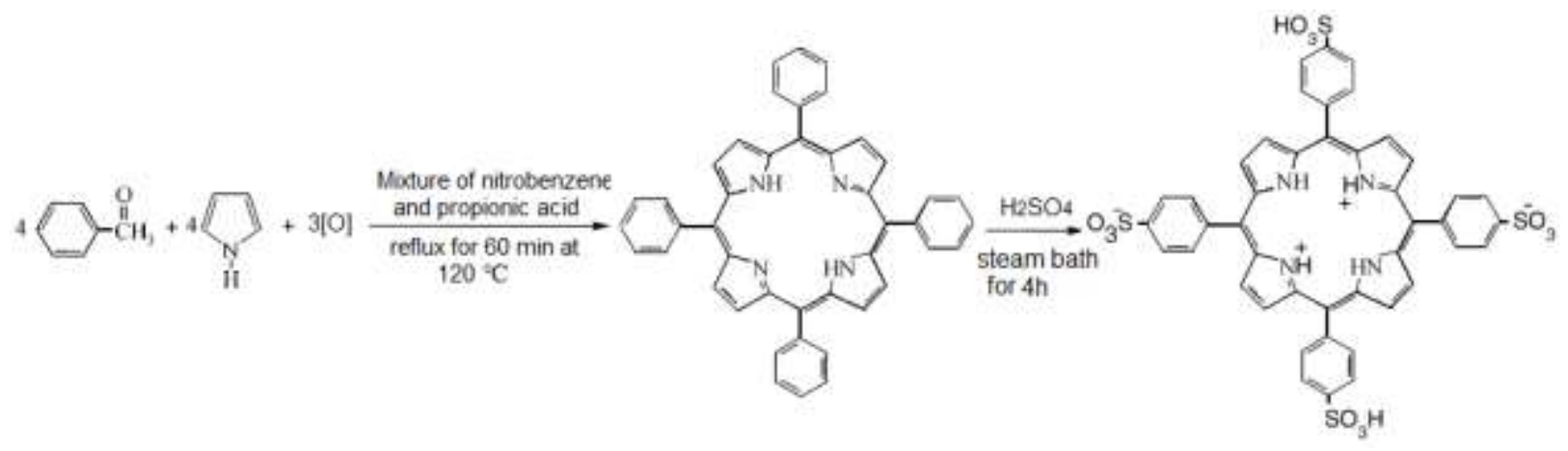

Scheme 1. Synthesis of the meso-tetrakis(4-sulfonatophenyl) porphyrin (TPPS). 


\subsection{Preparation of $\mathrm{NiO}$ nanoparticles}

The sol-gel method was employed as follows: $5 \mathrm{~g} \mathrm{Ni}\left(\mathrm{NO}_{3}\right)_{2} \cdot 6 \mathrm{H}_{2} \mathrm{O}$ and $5 \mathrm{~g}$ citric acid were used to prepare molar ratios from $\mathrm{Ni}\left(\mathrm{NO}_{3}\right)_{2} \cdot 6 \mathrm{H}_{2} \mathrm{O}$ :citric acid, 1:3. The precursor was dissolved in 100 $\mathrm{ml}$ pure alcohol until a clear mixed solution is formed; and then the solution was kept at $120{ }^{\circ} \mathrm{C}$ in an oven until the alcohol entirely evaporated and a green paste was obtained. After the sol-gel process, the paste was placed into a tube furnace, and calcined at $750{ }^{\circ} \mathrm{C}$ for $5 \mathrm{~h}$ in air.

\subsection{Modification of NiO nanoparticles by Ni-TPPS}

$0.02 \mathrm{mmol}$ of Ni-TPPS was dissolved in $50 \mathrm{ml}$ of $\mathrm{H}_{2} \mathrm{O}$ and $0.02 \mathrm{mmol}$ of finely grounded $\mathrm{NiO}$ nanoparticles $(2 \mathrm{mg})$ was added to this solution. The resulting suspension was stirred under refluxing for $5 \mathrm{~h}$ and then the solvent was removed under vacuum at room temperature. The resulting solution was cooled in room temperature for $24 \mathrm{~h}$. Consequently, the product was washed with ethanol until no porphyrin could be detected in the supernatant by UV-visible spectrophotometer.

\subsection{Photocatalysis procedure}

In a typical process, the catalytic reaction was carried out in a $100 \mathrm{ml}$ photoreactor (Scheme 2), which contain $50 \mathrm{ml}$ of $\mathrm{MB}$ dye $(20 \mathrm{mg} / \mathrm{l})$ solution and $0.05 \mathrm{~g}$ of catalyst. Before the irradiation, the solution was stirred in the dark $(15 \mathrm{~min})$ to allow equilibrium of the system. Irradiation was carried out using $400 \mathrm{~W}$ tungsten lamps as the light sources. All photocatalytic experiments were carried out at the same conditions. The distance between photoreactor and light sources was 20 $\mathrm{cm}$. Samples $(3 \mathrm{ml})$ were collected during the irradiation and MB solution were separated from the photocatalyst by centrifugation. The degradation was monitored by measuring the absorbance amount using a double beam UV-vis spectrophotometer (Shimadzu UV-1700) at $664 \mathrm{~nm}$ wavelength. 


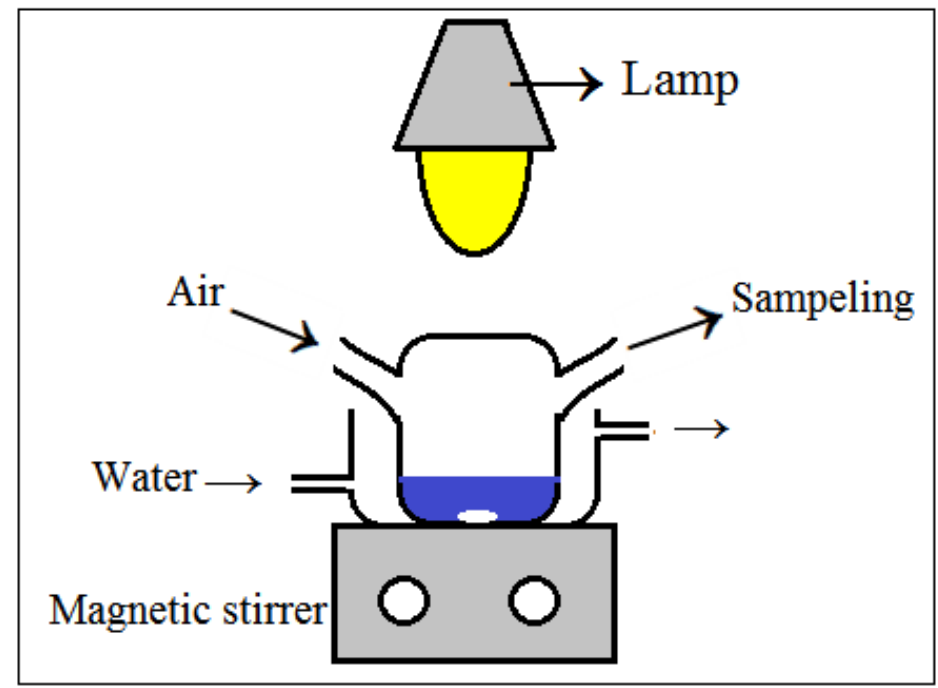

Scheme 2. Schematic representation of the photoreactor.

\section{Results and discussion}

\subsection{Morphological characterizations}

The morphology of the $\mathrm{NiO}$ particle was examined from SEM images, as shown in Fig. 1A. It was found that all synthesized $\mathrm{NiO}$ nanoparticles were quite uniform in size. Fig. 1B show SEM images of the TPPS/NiO nanoparticles.
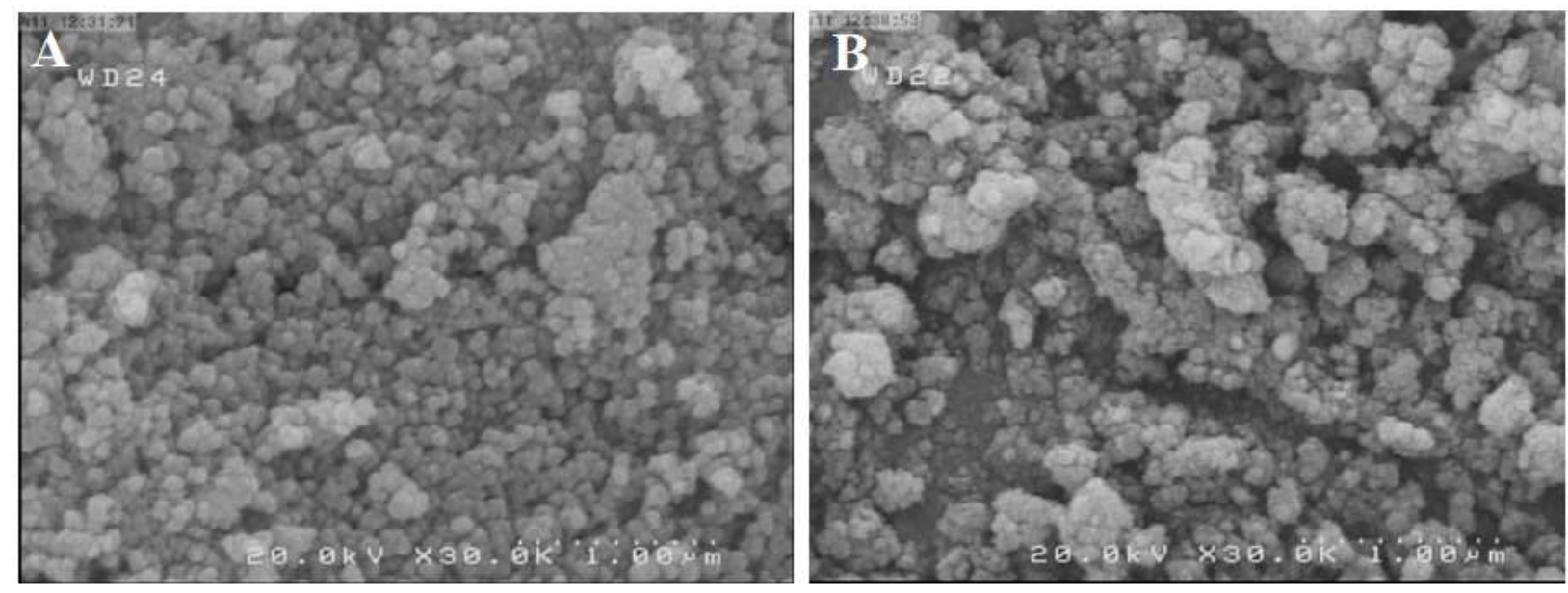

Fig. 1. The SEM images of the synthesized (A) NiO and (B) Ni-TPPS/NiO nanoparticles.

\subsection{The $X$-ray powder diffraction}

Fig. 2 shows the XRD pattern of $\mathrm{NiO}$ nanorod powders. It can be seen that all these peaks are in good agreement with cubic NiO (JCPDS Card, No. 00-001-1239). No peaks, any else phase of 
$\mathrm{NiO}$ or impurity peaks are observed, which indicates the high purity of the obtained $\mathrm{NiO}$ nanoparticles. On the whole, these diffraction peaks are sharp, narrow and symmetrical with a low and stable baseline, suggesting that the sample is well crystallized [19].

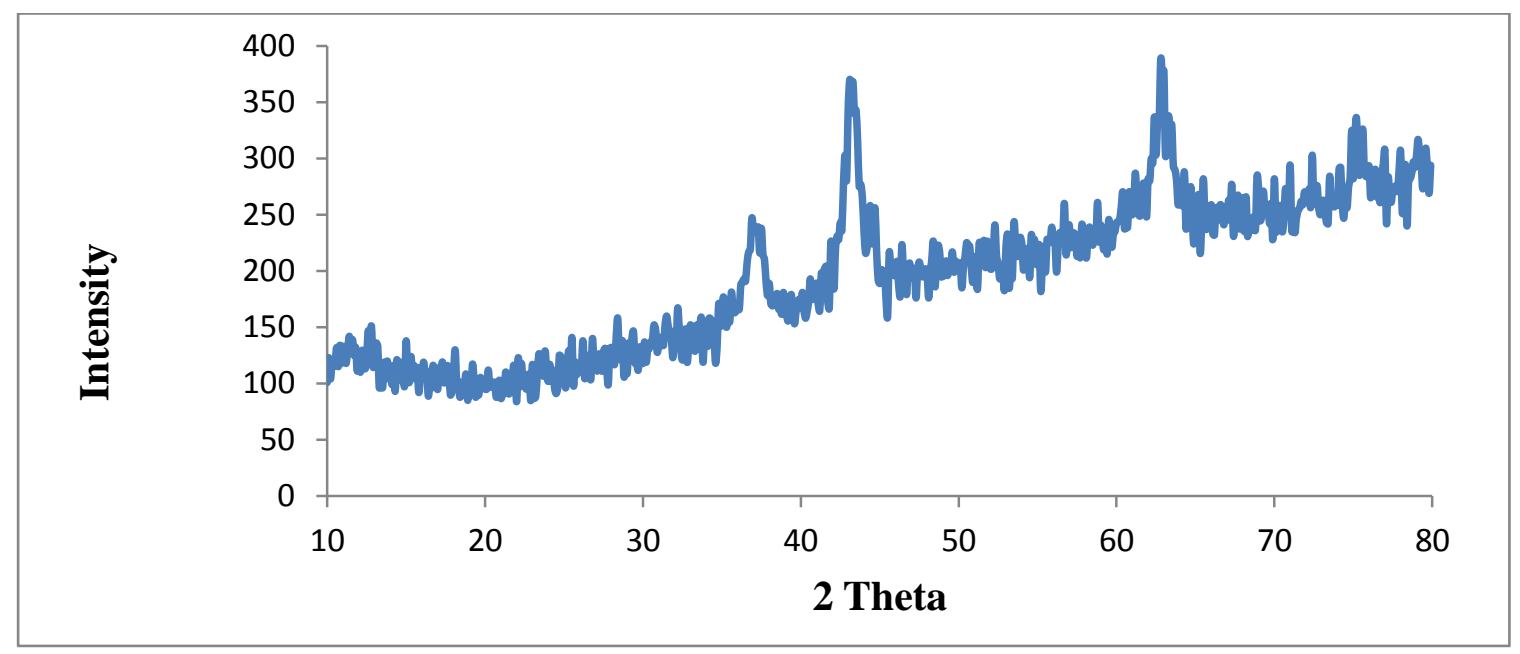

Fig. 2. The XRD pattern of the synthesized NiO nanoparticles.

\subsection{Optical properties}

The UV-vis diffuse reflectance spectra (DRS) of the synthesized $\mathrm{NiO}$ and TPPS/NiO nanoparticles are shown in Fig. 3A. The spectrum reveals a characteristic absorption peak of $\mathrm{NiO}$ at wavelength of 368 , which can be assigned to the intrinsic band-gap absorption of $\mathrm{NiO}$ due to the electron transitions from the valence band to the conduction band.

The absorption range of the composite is wider in comparison with those of pure TPPS and NiO nanoorods. The UV-visible spectrum of pure TPPS is containing a Soret peak in $413 \mathrm{~nm}$ and four Q peaks in 513, 553, 557 and $633 \mathrm{~nm}$. Besides, the Soret and Q bands of the porphyrin of the composite are red-shifted related to those of the pure TPPS. Furthermore, the intensity ratio of Soret band to $\mathrm{Q}$ bands of the TPPS/NiO is lower than that of the pure TPPS. Based upon these observations, it is demonstrated that the porphyrin has been assembled on the surface of $\mathrm{NiO}$ nanoparticles, and there exists a strong interaction between $\mathrm{NiO}$ nanoparticles and porphyrin in the TPPS/NiO. 


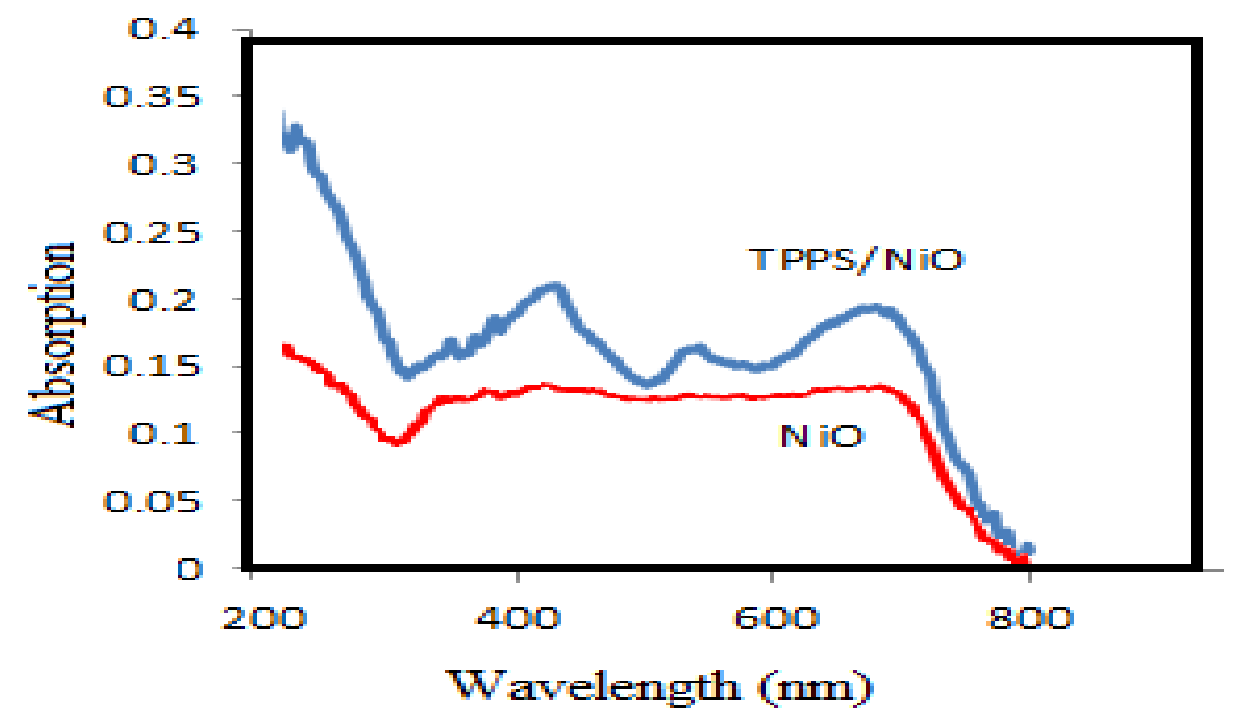

Fig. 3. The diffuse reflectance spectra (DRS) of the synthesized $\mathrm{NiO}$ and TPPS/NiO

\subsection{Fourier transforms infrared spectroscopy}

Fig. 4 shows the FT-IR spectra of the NiO nanoparticles, TPPS and the TPPS/NiO measured in the range of 390-4000 $\mathrm{cm}^{-1}$. The appearance of a sharp band at $450 \mathrm{~cm}^{-1}$ in the FT-IR spectra confirms the synthesis of $\mathrm{NiO}$ because it is the characteristic absorption band for the $\mathrm{Ni}-\mathrm{O}$ stretching vibration. Additionally broad absorption peaks centered at around $3446 \mathrm{~cm}^{-1}$ is caused by the $\mathrm{O}-\mathrm{H}$ stretching of the absorbed water molecules because the nanocrystalline materials exhibit a high surface to volume ratio.

For the FT-IR of TPPS, the stretching vibration of $=\mathrm{C}-\mathrm{N}$ and $-\mathrm{C}=\mathrm{N}$ bands (pyrrole) appeared at $1373 \mathrm{~cm}^{-1}$ and $1720 \mathrm{~cm}^{-1}$, respectively. The stretching asymmetric and symmetric vibration bands attributed to the $\mathrm{C}-\mathrm{H}\left(\mathrm{CH}_{2}\right)$ band are discernible in 2846, 2918 and $2939 \mathrm{~cm}^{-1}$. The appearance of the peaks at 890,1100 and $3387 \mathrm{~cm}^{-1}$ could be attributed to $\mathrm{C}_{6} \mathrm{H}_{4}$ (phenyl), $\mathrm{SO}_{3}$ group and $\mathrm{N}-\mathrm{H}$ band, respectively.

For the FT-IR of TPPS/NiO, the appearance of peaks corresponded to TPPS confirm that porphyrin have immobilized on $\mathrm{NiO}$ nanoparticles but these peaks are difficult to give precise values because of very low intensities due to a low loading of the macrocycle into the inorganic matrix. 


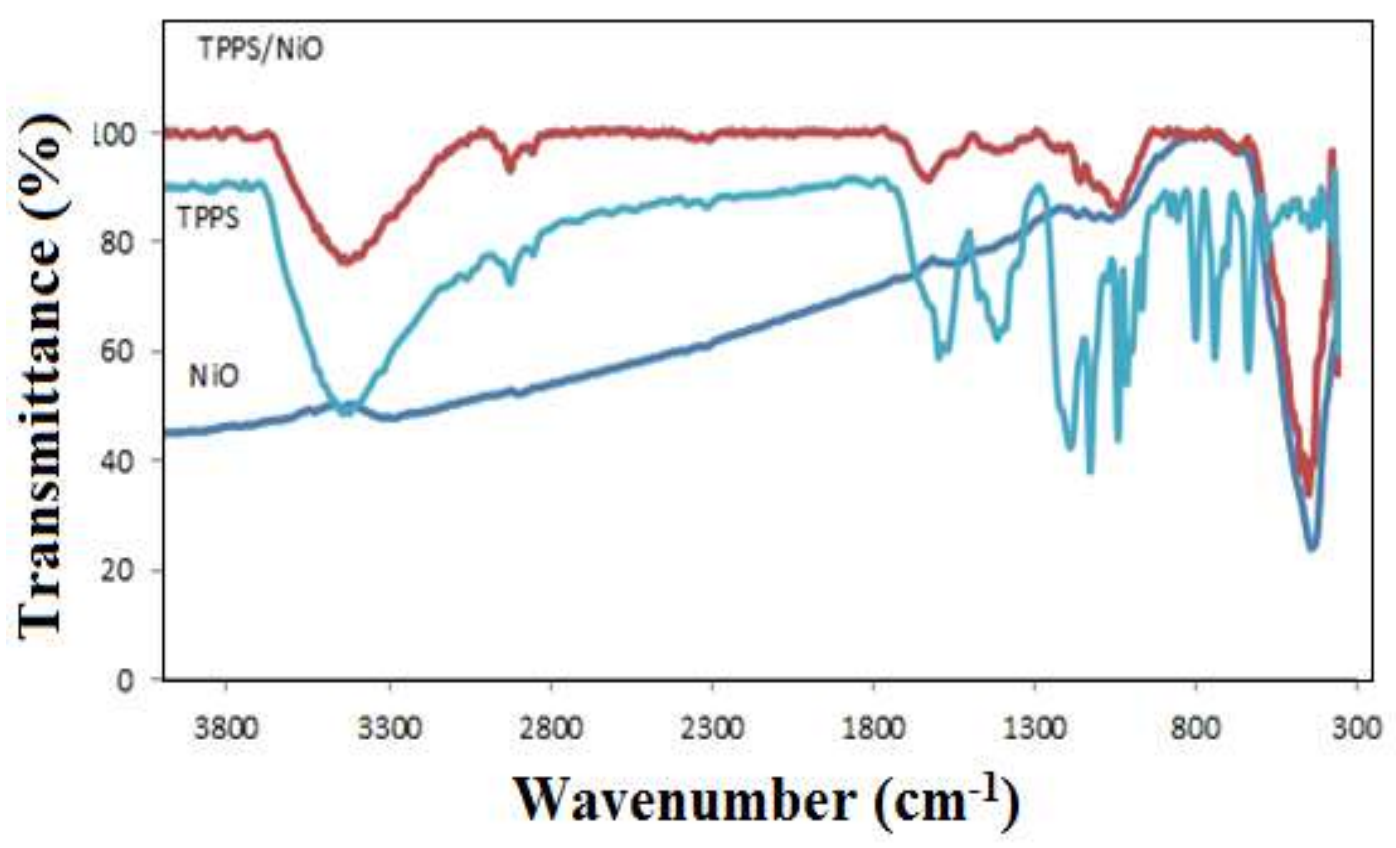

Fig. 4. The FT-IR spectrum of the synthesized NiO, TPPS/NiO and pure TPPS.

\subsection{Photocatalytic degradation of methylene blue}

The photocatalytic activities of as-synthesized two kinds of catalysts were evaluated by the degradation of organic dyes methylene blue in aqueous solution under light irradiation. The NiTPPS/NiO nanoparticles and $\mathrm{NiO}$ nanoparticles, with a high specific surface area, were used as photocatalysts for the decomposition of methylene blue by the superoxides and/or hydroxyl radicals formed at their interface. The characteristic absorption of MB at $664 \mathrm{~nm}$ was chosen to monitor the photocatalytic degradation process. Concerning the initial MB concentration, it can be concluded that there was a decrease in the photodegradation of MB with increasing initial MB concentration. Fig. 5 shows a typical photocatalytic degradation process of MB (initial concentration: $20 \mathrm{mg} / \mathrm{l}, 50 \mathrm{ml}$ ) using $0.05 \mathrm{~g} \mathrm{Ni}$-TPPS/NiO or $\mathrm{NiO}$ under visible light irradiation. The absorption peaks corresponding to $\mathrm{MB}$ diminished gradually as the exposure time was extended. 


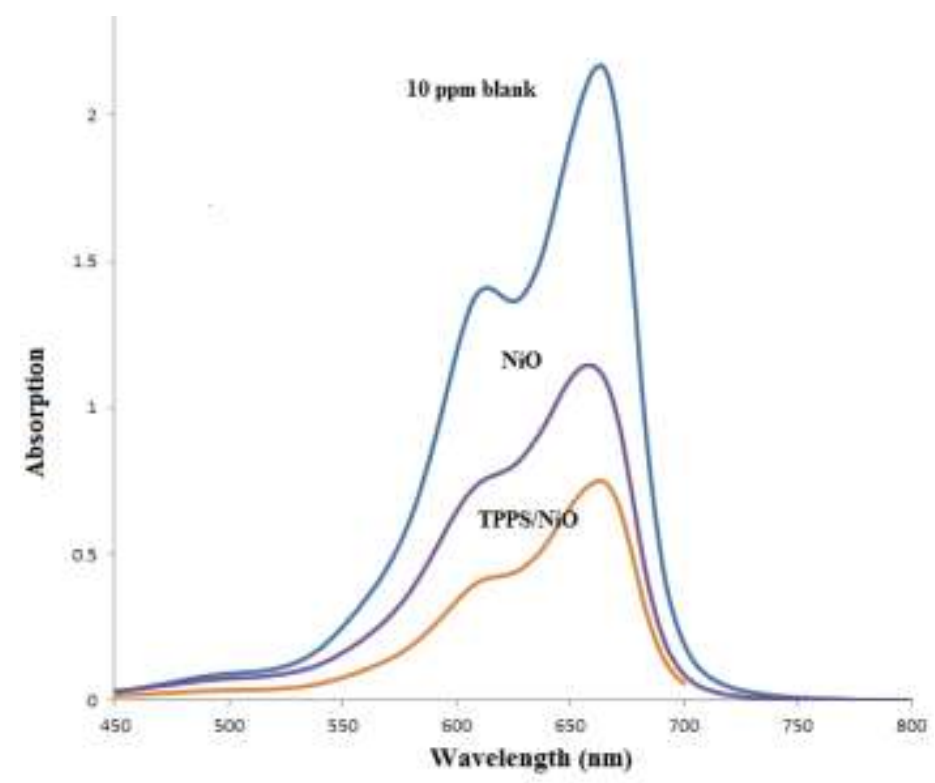

Fig. 5. The absorption spectra of the MB solution (initial concentration: $20 \mathrm{mg} / \mathrm{l}, 50 \mathrm{ml}$ ) in presence of TPPS/NiO and $\mathrm{NiO}$ catalyst $(0.05 \mathrm{~g})$ under visible light irradiation.

\section{Conclusion}

The photocatalytic degradation of $\mathrm{MB}$ in water was studied using $\mathrm{NiO}$ and visible light irradiation. The obtained results indicate that the photodegradation of MB was affected by the initial $\mathrm{MB}$ concentration and type photocatalyst. Ni-TPPS/NiO is better photocatalyst than $\mathrm{NiO}$ under visible light.

\section{References}

[1] Z. Mehraban, F. Farzaneh, A. Shafiekhani, Optical Materials 29 (2007) 927-931

[2] I. Willner, D. Mandler, Enzyme Microb. Technol. 11 (1989) 467.

[3] I. Willner, D. Mandler, J. Am. Chem. Soc. 106 (1984) 5352.

[4] I. Willner, D. Mandler, J. Chem. Soc., Chem. Commun. (1986) 851.

[5] I. Okura, N. Kaji, S. Aono, T. Kita, A. Yamada, Inorg. Chem. 24 (1985) 451.

[6] I. Okura, S. Kusunoki, S. Aono, Bull. Chem. Soc. Jpn. 57 (1984) 1184.

[7] H. Noriko, A. Yutaka, Energy Fuels 17 (2003) 1641.

[8] S. Yoshinobu, A. Yutaka, Bioconjug. Chem. 13 (2002) 898.

[9] H. Scheer, The Chlorophylls, CRC Press, Inc., Boca Raton, FL, 1991,p. 3. 